\title{
Large bowel infarct following antegrade scrotal sclerotherapy for varicocele: A case report
}

\author{
Patrizio Vicini, MD; Giovanni Battista Di Pierro, MD; ${ }^{\dagger}$ Pietro Grande, MD; ${ }^{\dagger}$ Giuseppe Voria, MD; \\ Gabriele Antonini, MD; Ferdinando De Marco, MD, ${ }^{*}$ Stefano Di Nicola, MD; Vincenzo Gentile, MD
}

"Department of Urology, "I.N.I." Italian Neurotraumatologic Institute Grottaferrata, Rome, Italy; †Department of Urology, "Sapienza" Rome University, Rome, Italy; §Department of Urology, S. Sebastiano Hospital Frascati, Rome, Italy

Cite as: Can Urol Assoc J 2014;8(9-10):e641-3. http://dx.doi.org/10.5489/cuaj.1822 Published online September 9, 2014.

\section{Abstract}

Varicocele is the main cause of male infertility. Treatment stops continuous damage to spermatogenesis, thereby potentially improving fertility. Among all the available procedures, the antegrade scrotal sclerotherapy (ASS), a combined radiological-surgical approach first introduced by Tauber, is gaining more popularity due to its minimal invasiveness. We report the case of a 35-year old man who was subjected to a colonic resection after antegrade scrotal sclerotherapy for varicocele. The procedure was necessary due to the embolization of venous anastomosis between the spermatic and mesenteric veins, which were not detectable at the preoperative phlebography.

\section{Introduction}

Varicocele represents the main cause of male infertility and leads to changes in testicular spermatogenesis in $60 \%$ to $70 \%$ of cases. Treatment prevents continuous damage to spermatogenesis, thereby potentially improving fertility. Successful treatment of varicocele improves semen quality in $40 \%$ to $60 \%$ of patients and recovers fertility in $10 \%$ to $40 \%{ }^{1}$ Among all the available procedures, the antegrade scrotal sclerotherapy (ASS) is a combined radiological-surgical approach first introduced by Tauber in $1988^{2}$ and is usually performed under local anesthesia. The procedure starts with a short longitudinal scrotal incision at the base of the scrotum, isolation of the funiculum and identification of the most enlarged vein. Thereafter, a small incision of the vein allows for the insertion of a 23-gauge needle to perform a venogram by iodine contrast. In the end atoxysclerol mixed with air is injected. ${ }^{2-5}$ This technique is well-established to treat varicocele. It is easier, faster and less invasive than open surgical and laparoscopic treatments..$^{5}$ It results in a persistence rate as high as $9 \%$ in adults and $3 \%$ in children. ${ }^{6}$ An analysis of seminal parameters showed a statistically significant improvement in the rate of fast progressive spermatozoa and reduction of immotile spermatozoa in patients who underwent ASS compared to open surgery. ${ }^{5}$ Reported complication rates are low and they include scrotal hematoma, sterile epididymitis (due to paravascular application of the sclerosing agent), testicular atrophy (accidental sclerotherapy of the testicular artery), as well as intestinal ${ }^{7}$ and abdominal wall necrosis (accidental sclerotherapy of the cremasteric artery). ${ }^{2}$

\section{Case report}

A 35-year-old man presented with severe oligoasthenospermia and diagnosed with third grade varicocele on ultrasound. After we explained the therapeutic options to the patient, he agreed to undergo antegrade scrotal sclerotherapy. Incising the base of the scrotum, the funiculum was identified and the most enlarged vein was isolated and suspended between two slack sutures. A little incision of the vein was performed to insert a 23-gauge needle. The right position of the needle was checked by washing the vessel lumen with saline solution; the iodine contrast was then injected to perform a venogram (Fig. 1). Finally, during the Valsalva manoeuvre, $1 \mathrm{~mL}$ of air was injected, followed by $3 \mathrm{~mL}$ of $3 \%$ ethoxysclerol (air-block technique).

Immediately after the injection of atoxysclerol, the patient reported intense pain in the left iliac fossa and flank and so the procedure was interrupted. Physical examination revealed an acute abdomen with positive Blumberg, abdominal distension and torpid peristalsis. A rectal probe was inserted and the patient was given prokinetics to facilitate intestinal peristalsis and steroids to reduce the inflammation.

A computed tomography (CT) scan revealed small aerial bubbles into mesenteric circulation of the sigmoid and 


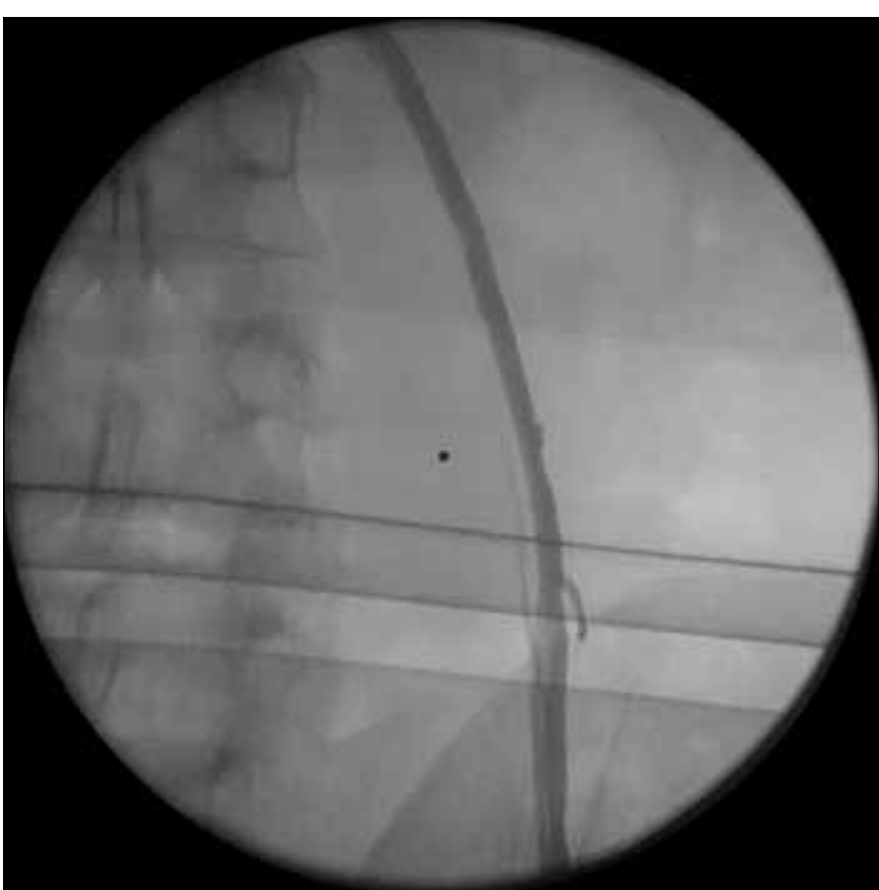

Fig. 1. lodine contrast injected to perform a venogram (not during the Valsalva manoeuvre).

descending colon (Fig. 2, Fig. 3). This finding was consistent with pneumatisation of the mesenteric venous plexus as a complication of the sclerotherapy and the tissues were minimally thickened with modest flogistic reaction of the loose tissue nearby. We decided to start anticoagulant therapy with low-molecular-weight heparin (LMWH) to prevent a potential venous thrombosis of the mesenteric circle secondary to chemical injuries.

Further CT scans were inconclusive, while the patient's pain continued to increase. The white blood cell count rose to $19 \times 10^{3} \mathrm{uL}$ the day after the procedure and continued to increase during the following days with a percentage of neutrophil granulocytes between $86 \%$ and $88 \%$.

As recommended by the general surgeon, a rectoscopy was performed, but it was inconclusive due to the presence of coagulated blood and mucus (Fig. 4). A new CT scan revealed a considerable aerial distension of the ileal and colic loops, mainly of the transverse and descending colon, with no radiocontrast outside the rectal ampulla.

Eight days after the procedure, the patient was immediately transferred to a general surgery unit due to severe pain resistant to morphine and benzodiazepine and concomitant septic shock. At this time, the CT revealed necrosis of the descending and sigmoid colon with faeces and air in the abdomen. A colic resection with end colostomy was carried out, with the rectum sewn shut and embeded in the pelvis for subsequent anastomosis (among transverse colon and rectum), which was performed 6 months later with complete fecal continence.

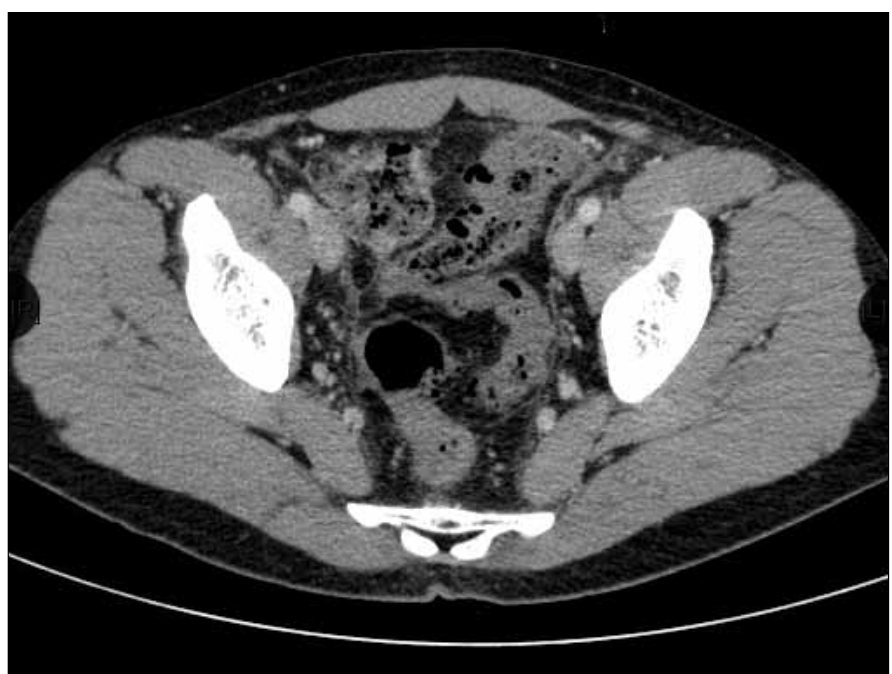

Fig. 2. Small aerial bubbles into mesenteric circulation of the sigmoid and descending colon.

\section{Discussion}

Although the existence of an anastomotic circle between the spermatic and mesenteric veins is not well-documented in the literature, Salerno and colleagues $^{8}$ suggest considering this finding as a risk for varicocele recurrence. Despite this, many authors do not routinely perform a preoperative phlebography ${ }^{9}$ before proceeding with sclerotherapy. Considered that on the phlebography the presence of communications between spermatic vein and visceral veins is reported in about $11 \%$ of patients, ${ }^{8}$ a complete angiographic study may represent a safer, if not compulsory, approach to guide the procedure, before antegrade sclerotherapy.

Indeed, the presence of an anastomotic circle should prompt a halting of the antegrade scrotal sclerotherapy to avoid injuries to the visceral vessels and should indicate surgical treatment. However, as previously reported by Fulcoli and colleagues, ${ }^{7}$ performing a phlebography is not devoid of possible false negative findings.

In the present experience, on the phlebography, internal spermatic vein was correctly visualized and there was no evidence of such anastomosis. lodine contrast was injected to perform a venogram (not during the Valsalva manoeuvre) (Fig. 1). ${ }^{2}$ The injection of $1 \mathrm{~mL}$ of air followed by $3 \mathrm{~mL}$ of $3 \%$ ethoxysclerol (air-block technique) during the Valsalva manoeuvre caused an increase in intravascular pressure due to the presence of air and to the Valsalva manoeuvre. These injections may have opened the venous passages between the spermatic and the inferior mesenteric veins that were not detectable by the phlebography.

Moreover, the injection of air followed by the sclerosing agent has the ability to better identify anastomotic circles between the spermatic and the inferior mesenteric veins compared to radiocontrast, due to the different densities 


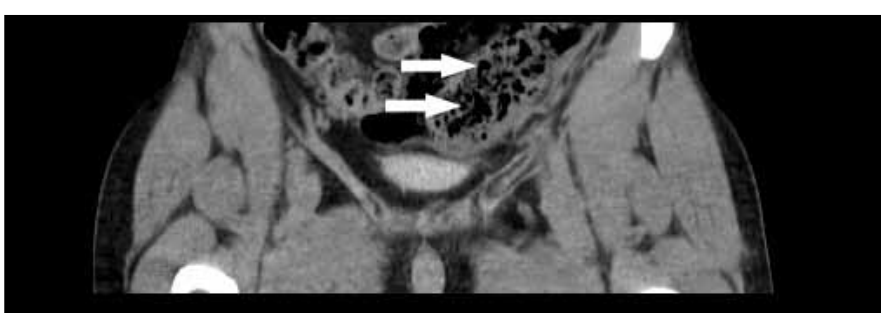

Fig. 3. Small aerial bubbles into mesenteric circulation of the sigmoid and descending colon.

of these 2 substances. Therefore, the cause leading to the necrosis of the descending and sigmoid colon was thought to be a bowel infarct secondary to venous thrombosis of the inferior mesenteric vein. On the other hand, given the small calibre of these vessels, no interventional radiology procedure could be performed to treat venous thrombosis. No further imaging or interventional radiology may have helped in the diagnosis, due to the variability of the vasculature and the technical difficulty. ${ }^{10}$

\section{Conclusion}

We found a complete angiographic study, mainly an antegrade phlebography, may represent a safer approach to guide the procedure before antegrade sclerotherapy. Therefore, performing a preoperative venogram during the Valsalva manoeuvre becomes it is imperative to reveal unknown anastomosis before there is more vascular damage. Although the Tauber approach represents a minimally invasive, welltolerated and easy to perform technique to treat varicocele, surgeons must be aware of the difficulties in determining possible anastomotic vessels between the intestinal and spermatic veins and possible severe side effects that may arise from its embolization.

Competing interests: Dr. Vicini, Dr. Di Pierro, Dr. Grande, Dr. Voria, Dr. Antonini, Dr. De Marco, Dr. Nicola and Dr. Gentile all declare no competing financial or personal interests.

This paper has been peer-reviewed.

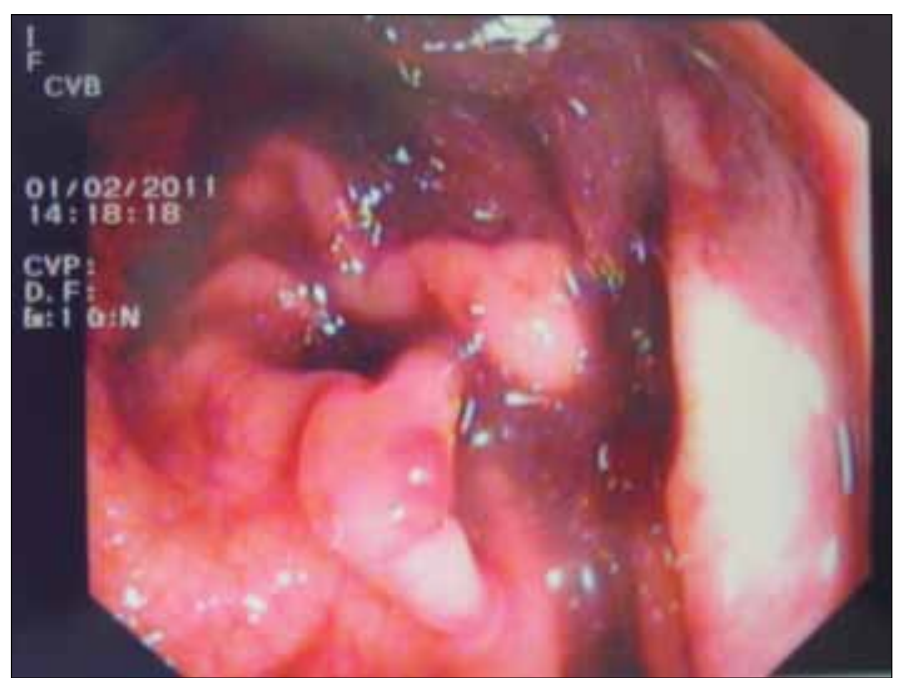

Fig. 4. The rectoscopy showing coagulated blood and mucus.

\section{References}

1. Green KF, Turner TT, Howards SS. Varicocele reversal of the testicular blood flow and temperature effects by varicocele repair. J Urol 1984;131:1208-11.

2. Tauber R, Pfeiffer D. Surgical atlas varicocele: Antegrade scrotal sclerotherapy. BJU Int 2006;98:1333-44. http://dx.doi.org/10.1111/i.1464-410X.2006.06579.x

3. Ficarra $V$, Porcaro $A B$, Righetti $R$, et al. Antegrade scrotal sclerotherapy in the treatment of varicocele: $A$ prospective study. BJU Int 2002;89:264-8. http://dx.doi.org/10.1046/j.1464-4096.2001.02418.x

4. Zaupa P, Mayr J, Höllwarth ME. Antegrade scrotal sclerotherapy for treating primany varicocele in children. BJU Int 2006;97:809-12. http://dx.doi.org/10.1111/i.1464-410X.2006.06033.x

5. Zucchi A, Mearini L, Mearini E, et al. Treatment of varicocele: Randomized prospective study on open surgery versus Tauber antegrade sclerotherapy. J Androl 2005;26:328-32. http://dx.doi.org/10.2164/ jandrol.04143

6. Johnsen $\mathrm{N}$, Johnsen I, Tauber R. Spermiogram findings following antegrade sclerosing of a varicocele. WMW 1997;147:81-3

7. Fulcoli $\mathrm{V}$, Costa $\mathrm{G}$, Gigli $\mathrm{F}$, et al. Ischemic necrosis of the sigmoid colon after antegrade sclerotherapy of idiopathic varicocele: a case report. Urologia 2013;80:162-4. http://dx.doi.org/10.5301/RU.2013.10722

8. Salerno S, Cannizzaro F, Lo Casto A, et al. Anastomosis between the left internal spermatic and splanchnic veins. Retrospective analysis of 305 patients. Radiol Med 2000;99:347-51.

9. Pecoraro G, Motta L, Olivio G, et al. Is phlebography crucial in Tauber varicocele sclerotherapy? Urologia 2008:75:105-7.

10. Artyukhin AA. Anatomy and microanatomy of the venous system of scrotal organs and spermatic cord. Bull Exp Biol Med 2007;143:99-104. http://dx.doi.org/10.1007/s10517-007-0027-9

Correspondence: Dr. Patrizio Vicini, Department of Urology, "I.N.I." Italian Neurotraumatologic Institute Grottaferrata, Rome, Italy; patrizio.vicini@gmail.com 